\title{
Activation of Toll-like receptor 9 attenuates unilateral ureteral obstruction-induced renal fibrosis
}

\author{
Bing-mu XIN, Xiao-xing WANG, Wen JIN, Hui-min YAN, Bing CUI, Xiao-wei ZHANG, Fang HUA, Hong-zhen YANG, Zhuo-wei \\ $\mathrm{HU}^{*}$
}

Molecular Immunology and Pharmacology Laboratory, Institute of Materia Medica, Chinese Academy of Medical Sciences \& Peking Union Medical College, Beijing 100050, China

\begin{abstract}
Aim: To study whether activation of TLR9 by CpG-ODN would protect against and/or reverse renal fibrosis.
Methods: Animals were treated with CpG-ODN before or after undergoing a unilateral ureteral obstruction (UUO) procedure. The interstitial fibrotic lesions of obstructed kidneys were evaluated using histology and immunohistostaining. The Th2-type cytokine profile and the expression and activity of sma and mad related protein (Smad)3, signal transducers and activators of transcription (Stat)3, extracellular regulated protein kinases (ERK), and p38 kinase were determined using RT-PCR or Western blot.

Results: The obstructed kidneys displayed a significant increase in interstitial fibrosis, an infiltration of macrophages in the interstitium, and an enhanced expression of Th2 cytokines. Prophylactic application of CpG-ODN (40 $\mu \mathrm{g} / \mathrm{kg}$ every 3 days from $2 \mathrm{~h}$ before UUO until the 14th day after UUO) suppressed the expression of $\alpha$-smooth muscle actin, collagen deposition, and hydroxyproline in the UUO kidneys of rats. Moreover, CpG-ODN not only decreased the infiltration of macrophages but also inhibited the expression of chemokines CCL2 and CCL5, the Th2 cytokine IL-13, and the profibrogenic cytokines transforming growth factor (TGF)- $\beta 1$ and plasminogen activator inhibitor (PAl)-1 in UUO kidneys of rats. Importantly, therapeutic administration of CpG-ODN (10 $\mu \mathrm{g} / \mathrm{mouse}$, ip, every 3 days from the 4th day to 21st day after UUO) reversed the established renal fibrosis, which was accompanied by significant reductions in the activity of ERK, Smad3, and Stat3 and an increase in the activity of p38 kinase.

Conclusion: The activation of TLR9 by CpG-ODN attenuates UUO-induced renal fibrosis by reversing an immunosuppressive microenvironment in the fibrotic renal tissue, which might be a novel therapeutic strategy against fibrotic renal diseases.
\end{abstract}

Keywords: inflammation; immunotherapy; renal fibrosis; Toll-like receptor 9; CpG-ODN; Th1/Th2; $\alpha$-smooth muscle actin; interferon- $\gamma$; transforming growth factor- $\beta 1$; plasminogen activator inhibitor-1

Acta Pharmacologica Sinica (2010) 31: 1583-1592; doi: 10.1038/aps.2010.202; published online 29 Nov 2010

\section{Introduction}

Renal fibrosis is a common pathologic pathway leading to end-stage renal failure (ESRF) of chronic nephropathy ${ }^{[1]}$. Emerging data indicate that chronic inflammation characterized by suppressive immune responses drives fibroproliferative disorders ${ }^{[2,3]}$. In particular, Th2-type cytokines contribute to the induction and maintenance of the chronic inflammatory response and play a critical role in the development of fibroproliferative lesions ${ }^{[4]}$, and Th1-type cytokines inhibit tissue scarring $^{[5]}$. Buffalo/Mna rats that have a spontaneous nephrotic syndrome in parallel with focal and segmental glomerular lesions have a Th2-dominant cytokine profile in their kid-

\footnotetext{
* To whom correspondence should be addressed.

E-mail huzhuowei@imm.ac.cn

Received 2010-07-01 Accepted 2010-11-03
}

neys ${ }^{[6]}$. Similar to the Th1/Th2 concept of T-cell activation, an M1/M2 paradigm has been proposed to function in the development and resolution of tissue fibrosis. M2 macrophages have the ability to efficiently inhibit M1-driven inflammation and promote the expression of profibrogenic molecules, such as transforming growth factor (TGF)- $\beta$, fibronectin, proline, and tissue inhibitor of metalloproteinases (TIMPs) ${ }^{[7,8]}$. Therefore, reversing suppressive immune responses may be a way to attenuate the progression of renal fibrosis.

Toll-like receptors (TLRs), as key pattern recognition receptors (PRRs) of the innate immune system, represent the first line of defense against infection and injury by responding to exogenous pathogen-associated molecular patterns (PAMPs) or endogenous damage-associated molecular patterns (DAMPs) released from damaged tissues or dying cells. The activation of TLRs enables antigen-presenting cells to mature 
and secrete cytokines and subsequently modifies and directs the ensuing adaptive immune response $\mathrm{e}^{[9]}$. TLR9 is primarily expressed in immune cells and recognizes bacterial DNA containing unmethylated $\mathrm{CpG}$ sequences. The activation of TLR9 results in the production of the Th1-type cytokine IL-12, which tends to direct a Th1-type immune response ${ }^{[10]}$. Synthetic oligodeoxynucleotides containing unmethylated CpG motifs (known as CpG-ODN) mimic the activity of bacterial DNA to activate TLR9. In macrophages, CpG-ODN stimulates an nuclear factor kappa B (NF-kB)-mediated expression of tumor necrosis factor (TNF)- $\alpha$ and co-stimulatory molecules, such as MHC class II, CD80, and CD86. In myeloid dendritic cells (DCs), CpG-ODN induces the maturation of DCs to elicit a Th1-dominant response characterized by the production of IL-12 and IFN- $\gamma$. Phase I/II clinical trials with a TLR9-based vaccine have already been initiated to reverse the underlying Th2-dominant immune dysregulation in allergic patients ${ }^{[11]}$. CpG-7909 is currently in phase III clinical trials to treat advanced non-Hodgkin's lymphoma, basal cell carcinoma, malignant melanoma, renal cell carcinoma, and cutaneous T-cell lymphoma ${ }^{[11]}$. Notably, the activation of TLR9 induces a type I IFN-dependent anti-inflammatory response in experimental colitis ${ }^{[12]}$. The role of TLR9 in renal diseases is complex. Previous studies have shown that the activation of TLR9 aggravated immune complex glomerulonephritis, but this role of TLR9 was assumed, as knockout mice were not used $^{[13]}$. In MRL/lpr mice, TLR9 deficiency increased disease activity, with mice displaying more severe skin lesions and greater scoring for glomerular and interstitial renal lesions compared with wild-type littermates ${ }^{[14]}$. However, the role of TLR9 in renal interstitial fibrosis has not been examined.

In this study, we hypothesized that the TLR9 agonist CpGODN might prevent and even reverse renal fibrosis through the induction of a consistent Th1-dominant response and the inhibition of a Th2-dominant immunoenvironment in kidneys. We found that CpG-ODN significantly suppressed renal fibrotic lesions by inhibiting the expression of Th2 and profibrogenic cytokines in unilateral ureteral obstruction (UUO) kidneys. Importantly, the therapeutic administration of CpG-ODN reversed the established renal fibrosis via reversal of the Th2-dominant immunoenvironment. These results suggest that TLR9 is involved in the process of healing renal injuries and that targeting TLR9 with TLR9 agonists to modulate immune responses might be effective in attenuating renal fibrosis after UUO.

\section{Materials and methods Reagents}

CpG-ODN (ODN1826: 5' -tcc atg acg ttc ctg acg tt-3', phosphorothioate linkage) and a control ODN without the CpG motif (nCpG-ODN: $5^{\prime}$-tcc atg agc ttc ctg agc tt-3', phosphorothioate linkage) were purchased from SBS Genetech (Beijing, China). Anti- $a$-smooth muscle actin (SMA), -interferon (IFN)- $\gamma$, -TGF- $\beta 1$, -interleukin (IL)-13, -plasminogen activator inhibitor (PAI)-1, -IL-17A, -CD68, and -GAPDH Abs were purchased from Santa Cruz Biotechnology (Santa Cruz, CA). Anti-E-cadherin was purchased from R\&D Systems (San Jose, CA). Antiextracellular regulated protein kinases (ERK), -p-ERK, -sma and mad related protein (Smad)3, -p-Smad3, -p38, -p-p38, and -signal transducers and activators of transcription (Stat)3 were purchased from Cell Signaling Technology Inc (Danvers, MA). Enhanced chemiluminescence plus Western blotting detection reagents were from Amersham Biosciences (Piscataway, $\mathrm{NJ}$ ). Hydroxyproline test kits were purchased from Nanjing Jiancheng Bioengineering Institute (Nanjing, China). All other materials were purchased from standard commercial suppliers.

\section{Animal experiments}

All animals were purchased from Beijing Vital River Lab Animal Technology Inc (Beijing, China) and housed under standard conditions for humidity, room temperature, and darklight cycles with water and food ad libitum. Animal studies were conducted following the principles outlined by the Institutional Committee for the Ethics of Animal Care and Treatment in Biomedical Research.

\section{Experiment 1}

Male Sprague-Dawley rats $(180 \pm 20 \mathrm{~g})$ were randomly divided into five groups: sham, UUO, IFN- $\gamma$-treated, CpG-ODNtreated and $n C p G-O D N$-treated $(n=8-10)$. UUO was performed as previously described ${ }^{[15]}$. In brief, anesthetized rats were subjected to left flank incisions, and UUO was performed by complete ligation of the left ureter at the upper one-third portions. The kidneys and ureters of sham rats were exposed but not ligated. IFN- $\gamma$-treated rats were ip injected with IFN- $\gamma$ $\left(100000 \mathrm{IU} \cdot \mathrm{kg}^{-1} \cdot \mathrm{d}^{-1}\right)$ from $2 \mathrm{~h}$ before UUO to the 14 th day after UUO. CpG-ODN-treated and nCpG-ODN-treated rats were ip injected with CpG-ODN or nCpG-ODN ( $40 \mu \mathrm{g} / \mathrm{kg}$ every 3 days) from $2 \mathrm{~h}$ before UUO to the 14th day after UUO. Both sham and UUO rats received the same volume of saline.

\section{Experiment 2}

Male C57BL/ 6 mice ( 8 weeks old, $18 \pm 2 \mathrm{~g}$ ) were randomized to sham, UUO, and CpG-ODN-treated groups $(n=13-15)$. CpGODN (10 $\mu \mathrm{g} /$ mouse) were ip injected every 3 days starting on the 4th day after UUO. Sham and UUO mice were treated with the same volume of saline $(0.1 \mathrm{~mL} / 10 \mathrm{~g})$. Animals were sacrificed on the 14th or 21st day after UUO.

\section{Histology and immunohistostaining}

Kidneys were fixed in $4 \%$ paraformaldehyde, embedded in paraffin and cut into $4-\mu \mathrm{m}$ paraffin sections. Changes in renal morphology and fibrosis were evaluated using H\&E and Masson trichrome staining. All immunohistologic studies were performed on paraffin-embedded sections as previously described $^{[16]}$. Renal inflammation and fibrosis were graded by a professional researcher of pathology, who was blinded to the experimental groups. The average integrated optical density (IOD) of the collagen area was determined using Image- 
Pro Plus image analysis software (Media Cybernetics, Silver Spring, MD) in 20 randomly chosen fields per tissue sample, at a magnification of 200 times.

For immunofluorescence analysis, the tissue was snapfrozen in optimal cutting temperature compound (Sakura FineTech, Tokyo, Japan) and cut into 10- $\mu$ m cryostat sections. The expression of a-SMA and E-cadherin was assessed using indirect immunofluorescence staining. Anti-a-SMA and antiE-cadherin Abs were used as primary Abs, and corresponding Alexa Fluor 594-conjugated goat anti-rabbit IgG and FITCconjugated goat anti-mouse IgG (Santa Cruz Biotechnology, Santa Cruz, CA) were used as secondary Abs. The sections were photographed with an E2000U confocal microscope and evaluated using Leica TCS SP2 software.

\section{Biochemical assays}

The hydroxyproline assay was performed as previously described $^{[16]}$. In brief, the kidneys were hydrolyzed with 2.5 $\mathrm{mol} / \mathrm{L} \mathrm{NaOH}$ at $120^{\circ} \mathrm{C}$ and $0.1 \mathrm{kPa}$ for $40 \mathrm{~min}$. After neutralization with hydrochloric acid, the hydrolysates were diluted with distilled water. The hydroxyproline content in the hydrolysates was assessed calorimetrically at $550 \mathrm{~nm}$. The values were represented as hydroxyproline content $(\mu \mathrm{g})$ per gram of kidney tissue.

\section{RT-PCR}

Total RNA was extracted from frozen renal cortexes using TRIzol reagent (Invitrogen, Shanghai, China). Reverse-transcriptase polymerase chain reaction (RT-PCR) was performed using a Mycycler thermal cycler and analyzed using $1.5 \%$ agarose gels. The values of the target genes were normalized to $\beta$-actin.

\section{Western blot}

After tissue homogenization, the extracted proteins were separated using 10\% SDS-PAGE and subjected to immunoblotting with specific antibodies, including anti- a-SMA, -E-cadherin, -ERK, -p-ERK, -Smad3, -p-Smad3, -p38, -p-p38, and -Stat3. Signals were detected using enhanced chemiluminescence plus Western blot reagents (Amersham Biosciences, Piscataway, NJ), according to the manufacturer's instructions.

\section{Flow cytometry analysis}

Single-cell suspensions from spleens and kidneys of the mice were obtained using previously described methods ${ }^{[16]}$. After depletion of the red blood cells using red cell lysis buffer (eBioscience), the macrophages were detected using antiF4/80-FITC Ab and anti-CD206-TRITC Ab using multicolor flow cytometer. The data were analyzed using CellQuest software (Becton Dickinson, California, CA).

\section{Statistical analysis}

Results were represented as mean \pm SEM. Comparisons among several groups were determined with one-way ANOVA using SPSS. $P<0.05$ was considered significant.

\section{Results}

\section{CpG-ODN protected UUO mice from renal fibrosis}

Compared to sham mice, UUO mice displayed significantly more collagen deposition in the interstitium of obstructed kidneys (Figures 1A and 1B). Pretreatment with CpG-ODN significantly reduced the UUO-induced deposition of collagen in the interstitium (Figures 1A and 1B). The renal fibrotic change was also assessed using a-SMA immunostaining for activated myofibroblasts, which are the major component of the intracellular matrix ${ }^{[17]}$. UUO resulted in a substantial increase in activated fibroblasts in the interstitium and renal tubules (Figures $1 \mathrm{~A}$ and 1D). In addition, CpG-ODN significantly reduced the UUO-increased hydroxyproline content (Figure 1C), the amount of activated fibroblasts (Figure 1D), and the expression of a-SMA mRNA (Figure 1E) in the kidney, suggesting that CpG-ODN treatment protected mice from UUO-induced renal fibrosis. CpG-ODN delayed the progress of pathologic morphological changes in the UUO kidneys, including thrombosis, bladder-like kidneys and necrosis. IFN- $\gamma$ is a typical Th1-type cytokine that has been extensively tested in basic and clinical studies to treat hepatic and pulmonary fibrosis. We found that IFN-ץ also reduced the UUO-induced deposition of collagen in the interstitium.

\section{CpG-ODN ameliorated the UUO-stimulated inflammatory responses}

The renal tissue of UUO rats exhibited severe tubulointerstitial macrophage accumulation compared with sham rats. Pretreatment with CpG-ODN significantly reduced CD68 ${ }^{+}$ macrophages in the interstitium of renal tissues (Figure 2A). The enhanced expression of CCL2 and CCL5 in the tubular epithelial cells of patients with chronic progressive kidney disease has been associated with the increased interstitial recruitment of inflammatory cells ${ }^{[16,18]}$. Therefore, we examined the expression of CCL2 and CCL5. UUO significantly enhanced the expression of CCL2 mRNA $(P<0.05$, Figure $2 \mathrm{~B})$ but not CCL5 mRNA (Figure 2C). Pretreatment with CpG-ODN significantly reduced the UUO-stimulated mRNA expression of CCL2 and CCL5 (Figure 2B and 2C). Both Th1 and Th2 cytokines were notably elevated in the kidneys of UUO rats (Figure 2 ). The expression of cytokines always occurred at tubular or interstitial sites beyond glomeruli. CpG-ODN significantly inhibited the UUO-upregulated expression of IL-13 (Figure 2D) but did not change the expression of IL-12 and IFN-Y (data not shown). TGF- $\beta 1$ is necessary for the development of the tubulointerstitial fibrosis caused by $\mathrm{UUO}^{[19]}$. We found that UUO resulted in strong immunostaining of TGF- $\beta 1$ in dilated and atrophic tubular sites (Figure 2E). Pretreatment with CpG-ODN markedly decreased the immunostaining intensity of TGF- $\beta 1$ (Figure 2E). PAI-1 is another important profibrogenic cytokine ${ }^{[20,21]}$, and PAI-1 ${ }^{-/-}$mice are deficient in the production of Th2 cytokines. CpG-ODN markedly reduced the UUO-enhanced expression of PAI-1 (Figure 2F). IL-17A is a cytokine secreted by Th17 cells and has been found to promote TGF- $\beta 1$-dependent pulmonary fibrosis ${ }^{[22]}$. The differentia- 
A
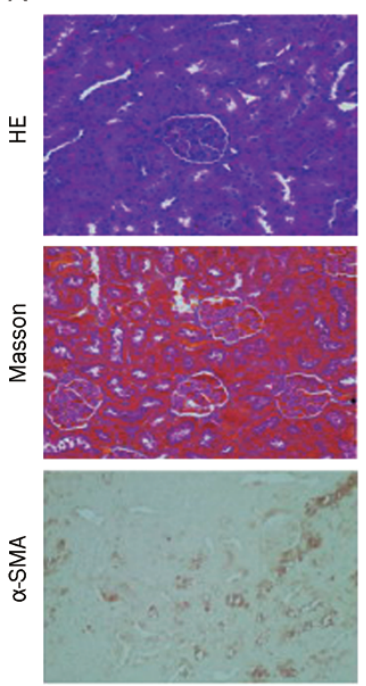

B

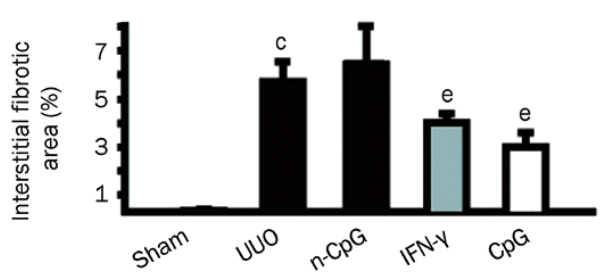

D

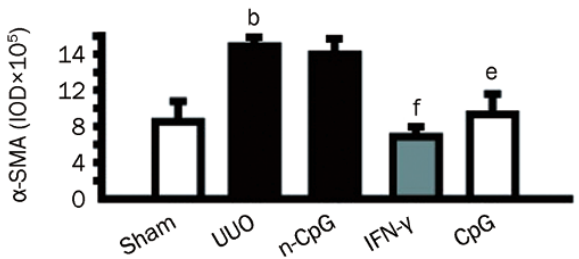

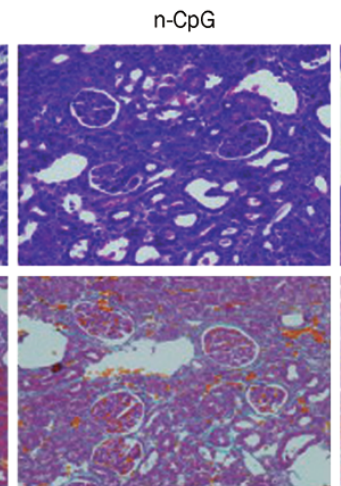
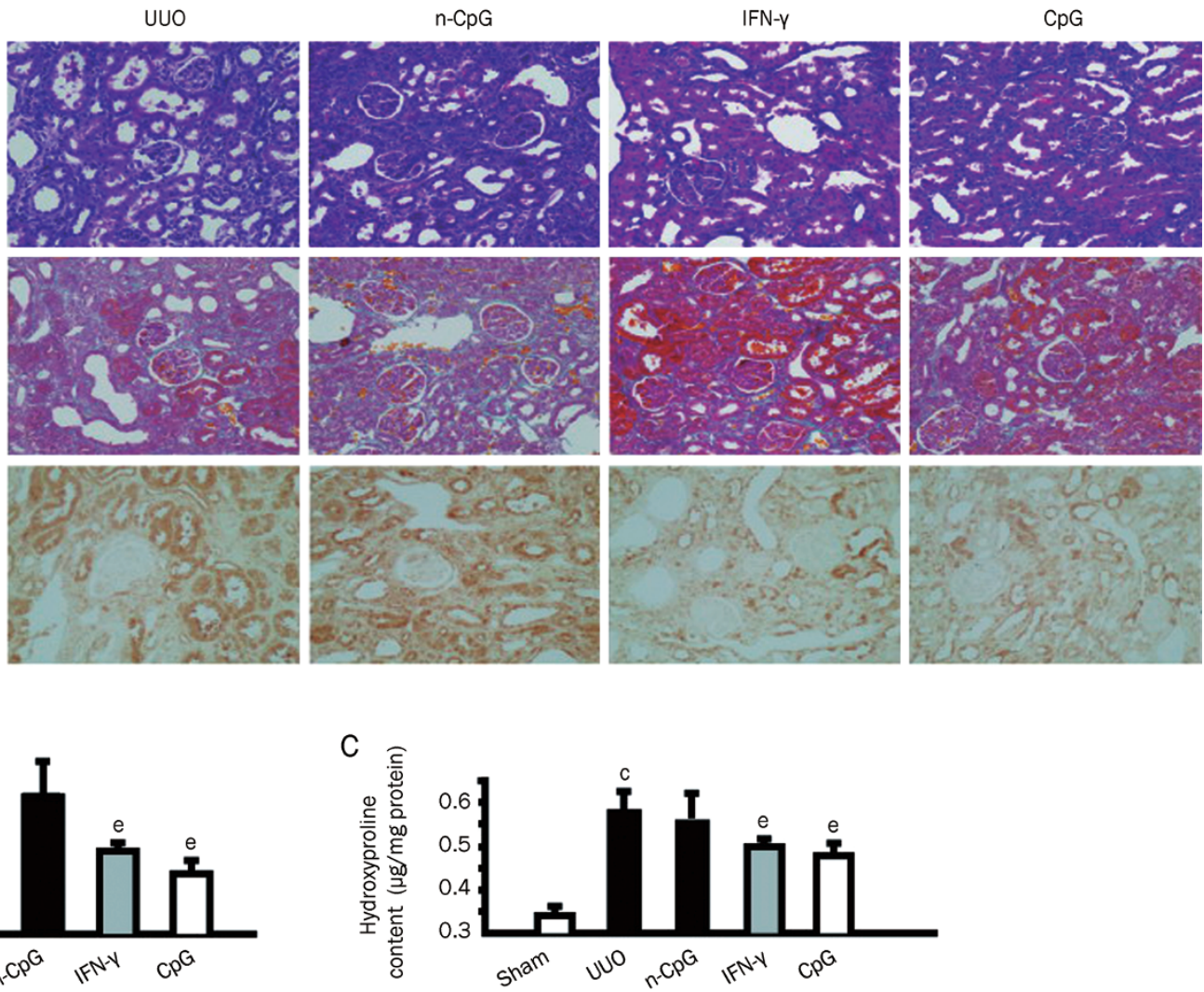

$\mathrm{E}$

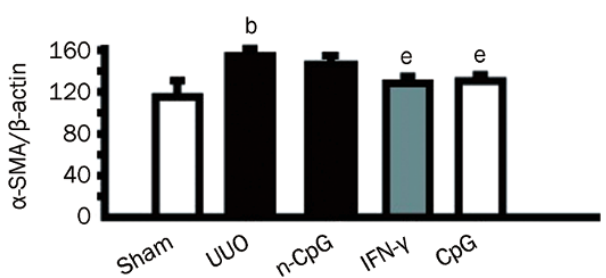

Figure 1. CpG-ODN prevented UUO mice from developing renal fibrosis. The rats received CpG-ODN or nonCpG-ODN (40 $\mu \mathrm{g} / \mathrm{kg}$ every 3 days) from $2 \mathrm{~h}$ before UUO until the 14th day after UUO. (A) Representative images of kidneys from sham, UUO, CpG-ODN (CpG), nonCpG-ODN (n-CpG) and IFN-y rats stained with H\&E, Massons and $\alpha$-SMA (magnification $\times 200$ ). (B) Summary of collagen area in renal tissue. (C) Hydroxyproline content in the kidney cortex. (D) $\alpha$-SMA expression in renal cortexes. (E) The mRNA expression of $\alpha-S M A$ in renal cortexes. Data are shown as mean \pm SEM $(n=6)$. ${ }^{b} P<0.05$, ${ }^{\mathrm{c}} P<0.01$ vs sham rats; ${ }^{\mathrm{e}} P<0.05,{ }^{\mathrm{f}} P<0.01$ vs UUO rats.

tion of naïve T cell to Th17 cells is modulated by TGF- $\beta 1$ and IL-6 ${ }^{[23]}$. We found that UUO resulted in a significant increase in the expression of IL-17A in the kidneys. Pretreatment with CpG-ODN markedly reduced the UUO-enhanced expression of IL-17A (Figure 2G). These results suggest that pretreatment with CpG-ODN ameliorates the UUO-stimulated Th2dominant inflammatory response.

\section{CpG-ODN reversed the established renal fibrosis}

Because pretreatment with CpG-ODN protected UUO rats from renal interstitial fibrosis by ameliorating the inflammatory response, we investigated whether CpG-ODN also had therapeutic efficacy in established renal fibrosis in mice. Indeed, we found that the obstructed kidneys from UUO mice showed a large collagen area compared with those from sham mice (Figure 3A, 3B, 3E). The collagen was mostly accumulated in the interstitium around tubules and glomeruli (Figure 3B). CpG-ODN significantly reduced the collagen area in the kidneys of UUO mice (Figure 3A, 3B, 3E). E-cadherin is a marker of epithelial cells. When epithelial-mesenchymal transition occurs, E-cadherin is downregulated in tubular epithelial cells ${ }^{[24]}$. A marked reduction in the expression of E-cadherin concomitant with a high expression of a-SMA was found in the obstructed kidneys from UUO mice. However, CpG-ODN treatment resulted in low-level expression of a-SMA, which was restricted to renal tubules and interstitial tissue (Figure 3C, 3F), and a high-level expression of E-cadherin in the renal tubules (Figure 3D, 3F). Notably, the protective effects of 
A

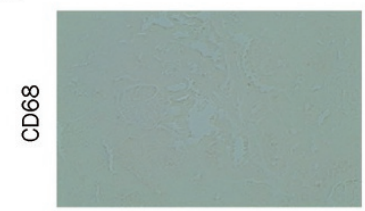

UUO

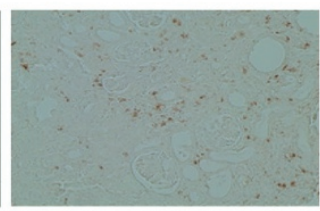

B
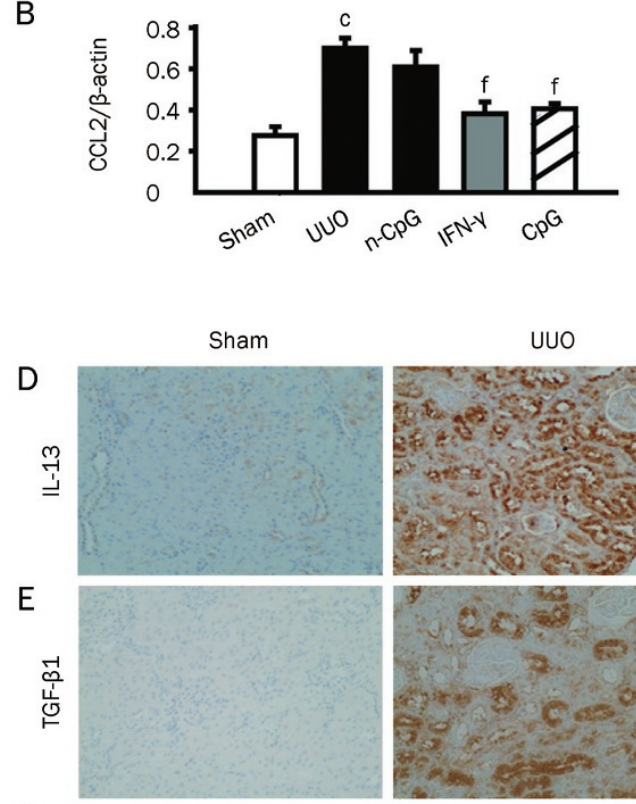

F

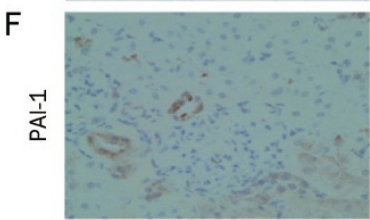

G

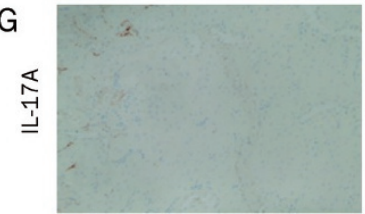

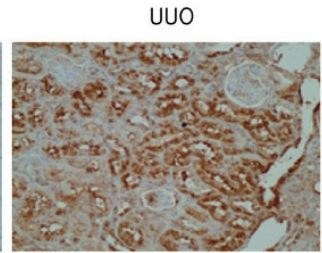
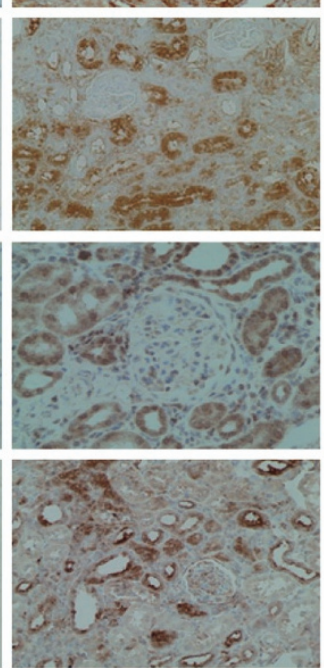
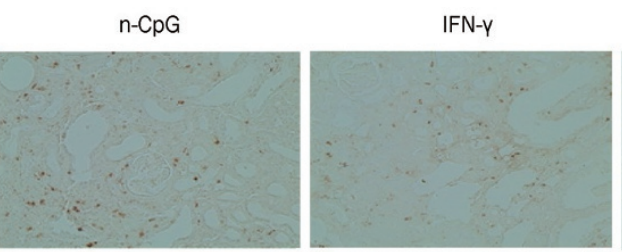

CPG

C
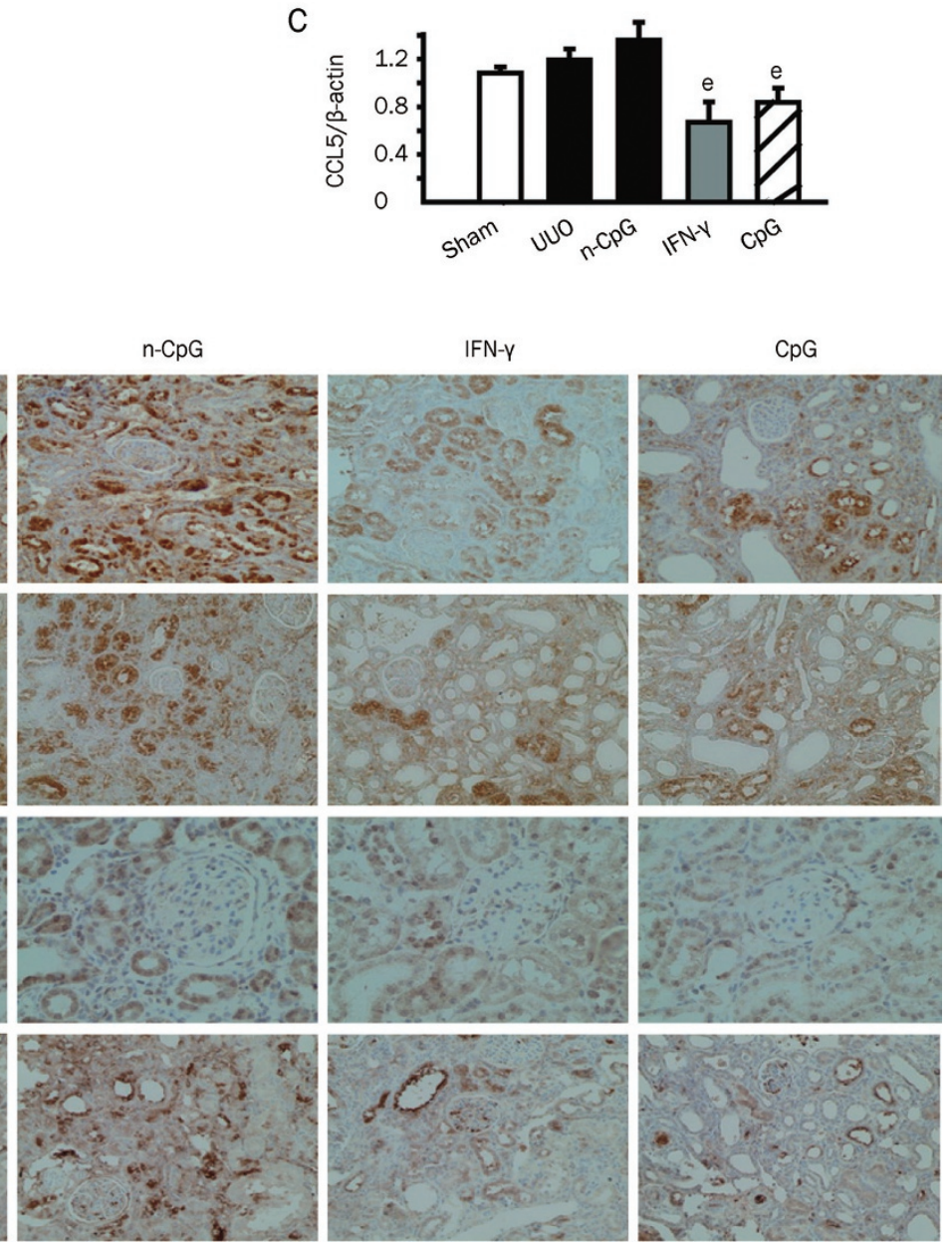

Figure 2. Prophylactic treatment with $\mathrm{CpG-ODN}$ reduced UUO-stimulated inflammatory responses in renal tissues. (A) The distribution of macrophages was evaluated using immunostaining with an anti-CD68 antibody. (B and C) The mRNA expression of CCL2 (B) and CCL5 (C) was analyzed using RTPCR. (D-G) The expression of IL-13 (D), TGF- $\beta 1(E)$, PAl-1 (F), and IL-17A (G) was detected using immunostaining, and the integrated optical density of the brown staining on renal tissue sections was semi-quantitatively evaluated using Image-Pro Plus image analysis software (Media Cybernetics, Silver Spring, MD). Data are shown as mean \pm SEM ( $n=6$ per group). ${ }^{b} P<0.05,{ }^{\circ} P<0.01$ vs sham rats; ${ }^{e} P<0.05,{ }^{f} P<0.01$ vs UUO rats.

CpG-ODN persisted up to 21 days after UUO (Figure 3G, 3J). Corresponding to reduced fibrotic lesions, the administration of CpG-ODN improved renal functions, as shown in Figure 4. There was a significant increase in the levels of serum creatinine and blood urea nitrogen (BUN) in UUO mice, whereas CpG-ODN treatment reduced the levels of serum creatinine and BUN (Figure 4). We theorized that with prolonged UUO, decompensation of contralateral renal tissue could have occurred, which would contribute to the decreased elimination efficiency of serum creatinine and urea in contralateral renal tissue. CpG-ODN attenuated UUO renal fibrosis and delayed decompensation of contralateral renal tissue.

\section{CpG-ODN reversed the immunosuppressive environment in} obstructed kidneys

Chronic inflammatory responses and excessive production of the profibrotic cytokines IL-13 and TGF- $\beta 1$ can turn a well-controlled healing response into a pathogenic fibrotic response $^{[2,3]}$. Treatment with CpG-ODN inhibited the UUOinduced expression of IL-13 (Figure 5A) but did not affect the expression of IFN-ץ (data not shown). In addition, CpGODN inhibited the UUO-enhanced expression of TGF- $\beta 1$ and 

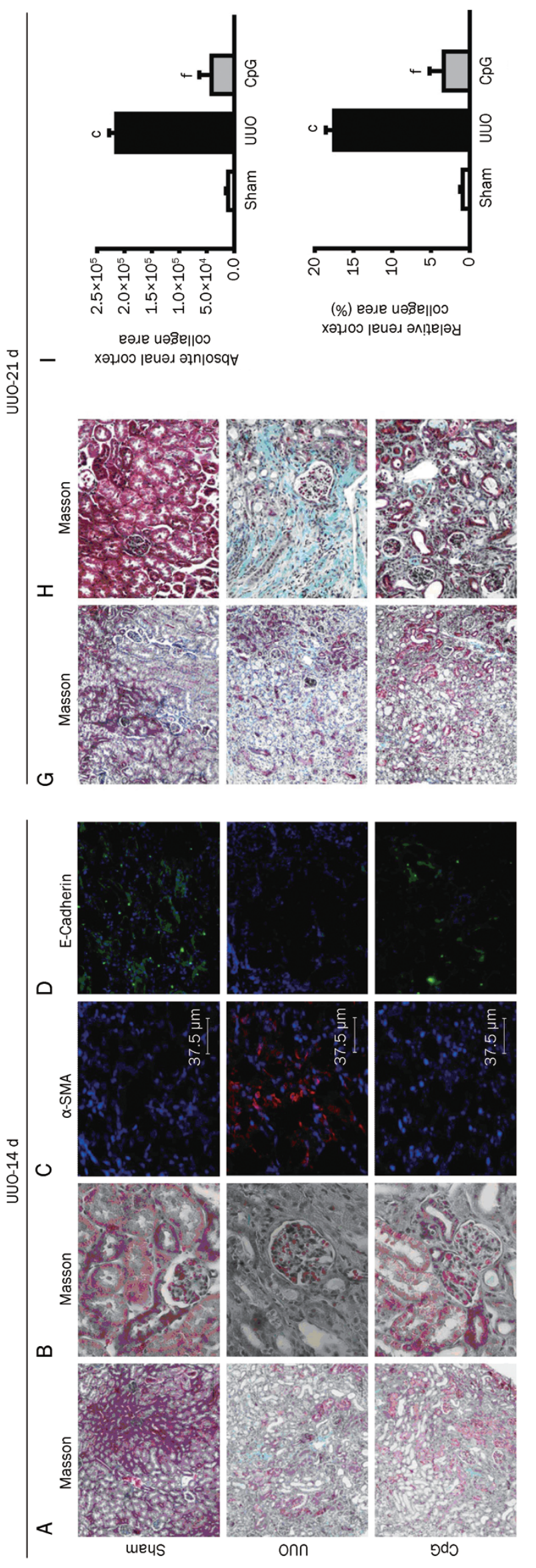
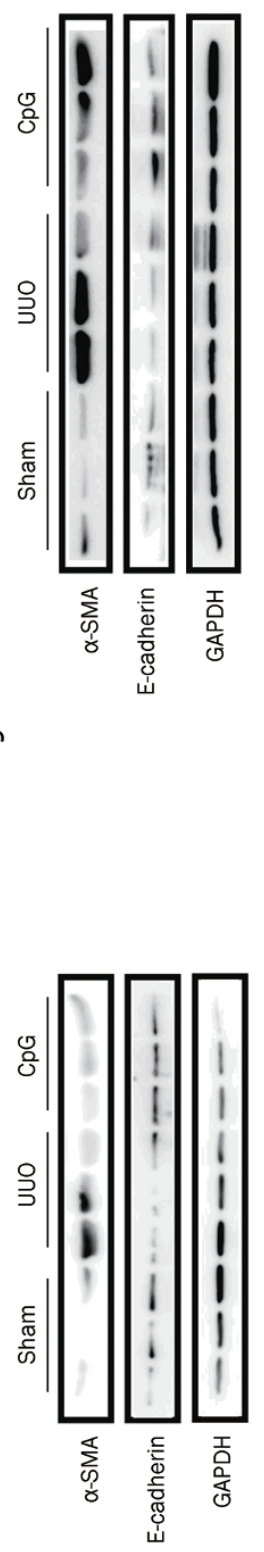

ᄂ

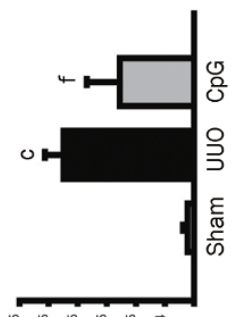

等

ш

еәле иәริе॥о0

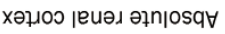
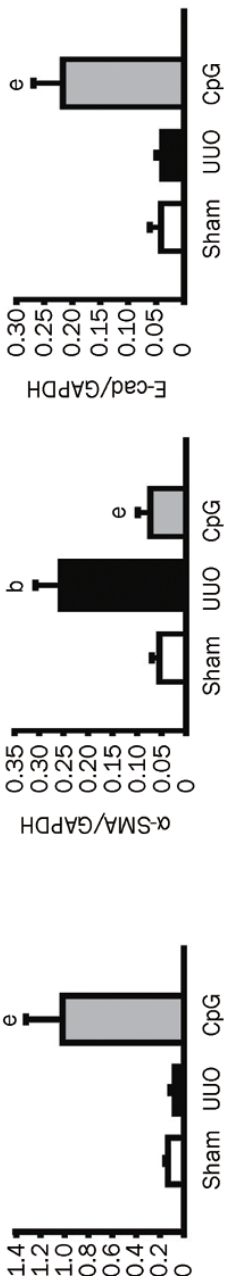

HOd $\forall 9 /$ peo-

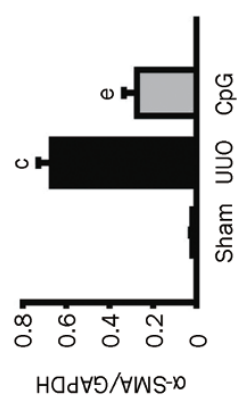

这高旁蓠

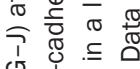

ज割

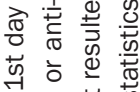

ते 0

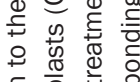

등 궁

๖ 흥 웅

ह हे ठํ워

원에

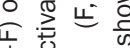

过

तิ

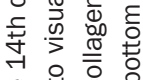

운 웅

可

表文造

类 क人

ह

is

วิ

i 을

용

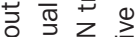

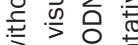

3 엉

응 응

势过要

造 $x^{2}$

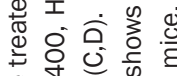

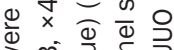

舫

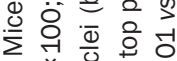

ค่

爻顿

塎

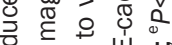

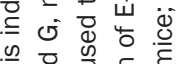

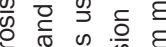

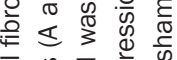

त्र

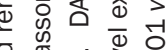

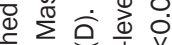

क्.

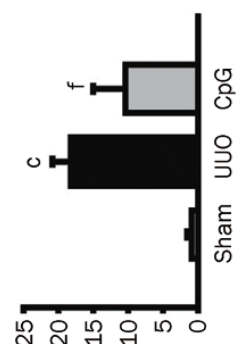

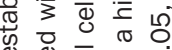

ه 항

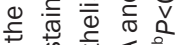

o क

क्षे

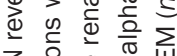

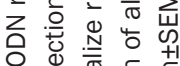

인

응 ब

लं 은 음

(\%) еәле иәвิе॥о хәдоо еиәд әк!ฺерәу

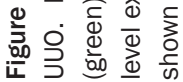


A
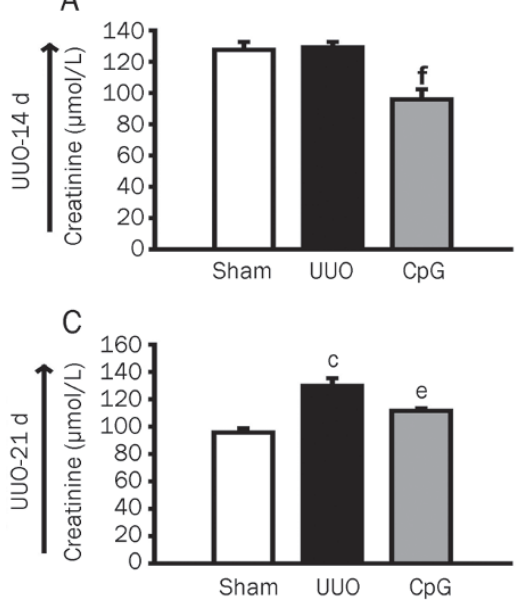

B

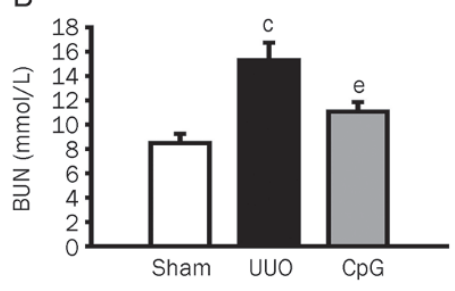

D

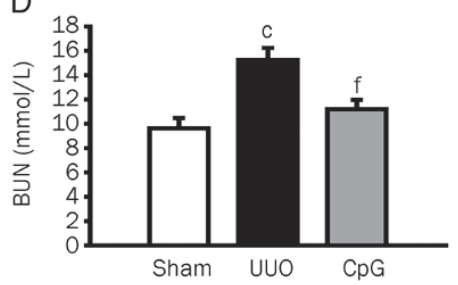

Figure 4. Therapeutic administration of CpG-ODN improved the functions of fibrotic kidneys. Kidney functions were evaluated by measuring the levels of serum creatinine and urea nitrogen (BUN). (A, C) CpG-ODN decreased the level of serum creatinine on day 14 (A) and day 21 (C) after UUO. (B, D) CpG-ODN decreased the level of urea nitrogen on day 14 (B) and day 21 (D) after UUO. Data are shown as mean \pm SEM ( $n=12-15) .{ }^{\mathrm{b}} P<0.05,{ }^{\mathrm{c}} P<0.01$ vs sham mice; ${ }^{\mathrm{e}} P<0.05,{ }^{\mathrm{f}} P<0.01$ vs UUO mice. alleviated fibrotic lesions (Figure 5A). The transcription factor Smad3 mediates TGF- $\beta 1$-induced profibrotic effects ${ }^{[15,25]}$. UUO significantly enhanced the phosphorylation of Smad3, although UUO did not increase the expression of Smad3 in the kidney. CpG-ODN treatment reduced the UUO-induced phosphorylation of Smad3 (Figure 5B). The transcription factor Stat3 is also involved in the regulation of Th1/Th2 responses; it enhances the production of immunosuppressive cytokines while reducing Th1 cytokines. We found that CpGODN inhibited the UUO-enhanced expression of Stat3, which was consistent with the change in Th2 immune responses (Figure 5C). Additionally, CpG-ODN repressed the UUO-induced phosphorylation of ERK and enhanced the phosphorylation of p38 (Figure 5D, 5E). Additionally, CpG-ODN treatment decreased the UUO-increased amount of F4/80 CD206 ${ }^{+} \mathrm{M} 2$ macrophages in the spleen (data not shown).

\section{Discussion}

Renal fibrosis can result from a wide variety of insults, including infection, oxidative stress, chemotherapeutic agents, and obstructive nephropathy. These stimuli induce and sustain the tissue wound-healing process ${ }^{[3]}$. The host's response to these insults, which is primarily mediated by the innate immune system, is intricately directed toward the recognition of PAMPs or DAMPs through PRRs, such as TLRs. The interaction of PRRs with PAMPs or DAMPs leads to the recruitment of inflammatory cells and the subsequent accumulation of extracellular matrix, as a consequence of chronic epithelial injury and the failure of repair because of aberrant epithelialmesenchymal interactions ${ }^{[8,26,27]}$. Indeed, our current study demonstrates that UUO stimulates a significant increase in the renal tissue-infiltrating M2 cells, which is correlated with the degree of renal fibrosis ${ }^{[2]}$. The key features of fibroproliferative diseases, including renal fibrosis, are distinctive cytokine profiles with elevated levels of immunosuppressive and/or profibrotic Th2, Th17, and Treg cytokines. These cytokines promote the activation and differentiation of fibroblasts to a more active cell type, the myofibroblast, to stimulate collagen production and deposition ${ }^{[8]}$.
Thus, the strategy to regulate suppressive immune responses using immune stimulants may prevent and reverse renal fibrosis. In particular, strategies directed toward eliminating immunosuppressive cells and cytokines might prove highly beneficial in the context of tissue fibrosis during chronic inflammation because their elimination would presumably diminish the concomitant chronic inflammatory and fibrotic mechanisms. Indeed, IFN- $\gamma$, a classical Th1 cytokine, has been widely used in the treatment of various tissue fibrotic diseases, such as pulmonary fibrosis, liver fibrosis, and renal fibrosis ${ }^{[28,29]}$. In our study, we found that IFN- $\gamma$ was an effective Th1-typical cytokine to improve renal fibrosis in a UUO model, which is in agreement with previous studies ${ }^{[30]}$. We have recently found that the BCG vaccine and administration of a TLR4 agonist individually protect experimental hypertensive mice from pressure overloadinduced cardiovascular fibrosis via regulation of the cardiovascular immune microenvironment ${ }^{[31]}$. Moreover, targeting TLR2 using an anti-TLR2 antibody or by knocking out TLR2 protects against and reverses progressive pulmonary fibrosis through the reversal of the lung injury-induced immunosuppressive environment ${ }^{[32]}$. In the current study, we found that activation of TLR9 by a systemic administration of CpG-ODN prevented and reversed UUO-induced renal fibrosis through the inhibition of Th2-dominant responses caused by the UUO procedure. Indeed, CpG-ODN-stimulated immune responses are beneficial in animal models for the treatment of a variety of diseases, such as cancers, allergy, and asthma, which demonstrates that CpG-ODN may represent a targeted immune modulator with a broad range of potential applications ${ }^{[11,33]}$.

Determining the mechanism responsible for the renoprotective role of the TLR9 agonist is complicated. It has been proposed that the activation of TLR9 can generate either suppressive or stimulatory responses ${ }^{[34,35]}$. TLR9 activation by CpG-ODN is beneficial to the resolution of renal inflammation and fibrosis. For example, CpG-ODN given prior to dextran sulfate sodium treatment had a potentially therapeutic effect in a rodent model of experimental colitis induced by dextran sulfate sodium because TLR9 triggered type I IFN, which 

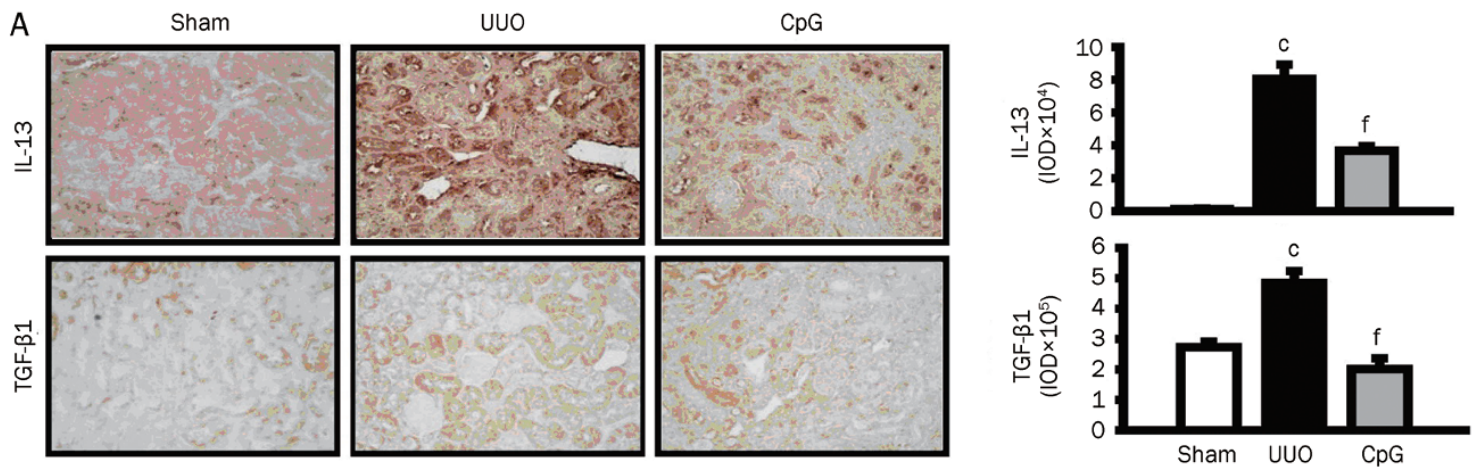

B
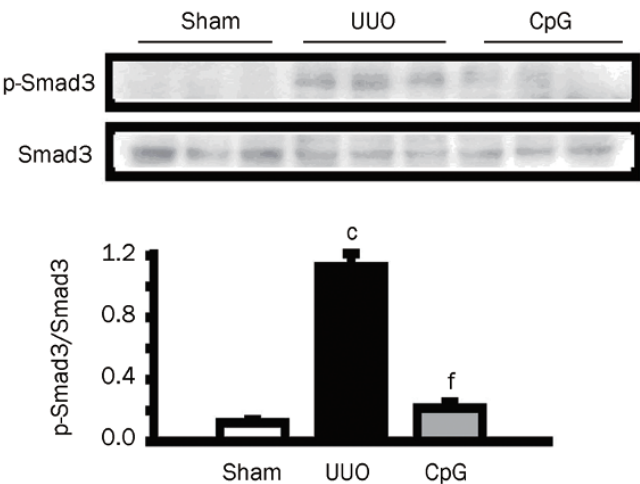

D
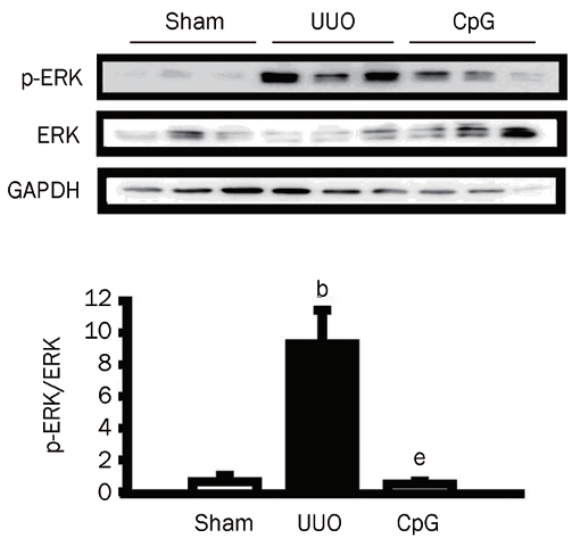

C

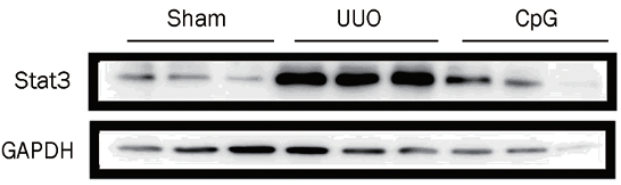

E
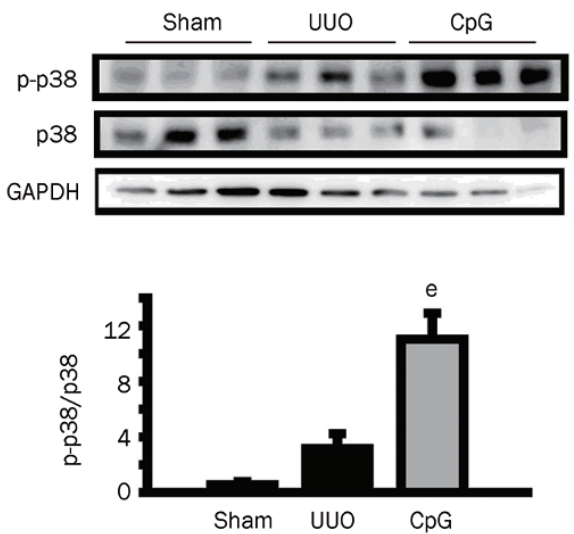

Figure 5. CpG-ODN reversed the immunosuppressive environment in the fibrotic renal tissue. (A) CpG-ODN reduced the expression of IL-13 (top panel) and TGF- $\beta 1$ (bottom panel). (B) CpG-ODN inhibited the phosphorylation of Smad3. (C) CpG-ODN downregulated the expression of Stat3. (D) CpG-ODN blunted the activity of ERK. (E) CpG-ODN enhanced the activity of p38. (B-E) The top panel shows a representative Western blot, and the bottom panel presents the summarized results. Data are shown as mean \pm SEM $(n=3) .{ }^{b} P<0.05,{ }^{\mathrm{c}} P<0.01$ vs sham mice; ${ }^{\mathrm{e}} P<0.05,{ }^{\mathrm{f}} P<0.01$ vs UUO mice.

has an anti-inflammatory effect ${ }^{[12]}$. Indeed, we found that both vaccination and therapeutic treatment with CpG-ODN blunted the severe inflammatory response and reversed the established fibrosis in obstructed kidneys, shifting the Th1/ Th2 balance in renal tissue toward a Th1 response. This shift was represented by the decreases in the expression of Th2 cytokines and pro-fibrotic factors PAI-1 and TGF- $\beta 1$, and the activity of ERK, Stat3, and Smad3 that regulate Th2-dominant immune responses ${ }^{[36-38]}$. However, there are contradictory reports that indicate that TLR9 activation aggravates inflammatory damage and related diseases. For example, in MRL lpr/lpr mice, CpG-DNA markedly enhanced the expression of CCL2 and CCL5 in nephritic kidneys, which contributes to the aggravation of glomeruli and tubulointerstitial damage ${ }^{[39]}$. Furthermore, TLR9 stimulation by CpG DNA caused Th1 polarization and exacerbated IgA nephropathy ${ }^{[13]}$. We believe that the effects of CpG-ODN on fibrotic processes might depend on the types and etiologies of different renal diseases. 
In contrast to the systemic autoimmunity of MRL lpr/lpr mice in which auto-antibodies to nucleic acid-associated antigens trigger glomerulonephritis, the UUO model recapitulates glomerular lesions of kidney disease in an accelerated manner by simulating the process of destructive renal interstitial fibrosis. Additionally, Th1 predominance in MRL/lpr mice and patients with increased levels of IgA has been repeatedly confirmed, which makes it likely that CpG-ODN induces the progression of renal disease. However, in our studies, the obstructed kidneys displayed significant interstitial fibrosis and a considerable upregulation of Th2 cytokine expression. CpG-ODN apparently inhibited the fibrogenic action in UUO renal tissue, which was attributed to a CpG-ODN-induced Th1-dominant response.

In summary, CpG-ODN not only significantly protects mice from UUO-induced renal fibrosis but also alleviates established renal fibrosis by reversing the tissue injuryinduced immunosuppressive environment in UUO kidneys. Our studies suggest that TLR9 is a promising target for the development of therapeutic agents against renal fibrosis and that eliminating immunosuppressive cells and factors using immunostimulants such as TLR9 agonists is a novel strategy for fibroproliferative diseases. Of course, one should be cautious in assuming an antifibrotic role for CpG-ODN in light of its proinflammatory activity. The role of TLR9 in renal fibrosis requires further study.

\section{Acknowledgements}

This study was supported by grants from the National Major Basic Research Program of China (2006CB503808), the National Natural Scientific Foundation (30672468; 30901814) and the Creation of Major New Drugs (2009ZX09301-00313). Dr Zhuo-wei HU is also supported by the Cheung-Kong Scholars Program of the Ministry of Education and by a Senior Oversea Chinese Scholar Fund from the Ministry of Personnel of China.

\section{Author contribution}

Bing-mu XIN designed and performed most of the experiments, analyzed and interpreted data and contributed to writing the manuscript; Xiao-xing WANG, Wen JIN, Hui-min YAN, Bing CUI, Xiao-wei ZHANG, Fang HUA, and Hongzhen YANG performed and analyzed individual experiments; and Zhuo-wei HU conceived the project, designed and interpreted experiments and wrote the manuscript.

\section{References}

1 Eddy AA. Molecular basis of renal fibrosis. Pediatr Nephrol 2000; 15 : 290-301.

2 Wynn TA. Fibrotic disease and the $\mathrm{T}(\mathrm{H}) 1 / \mathrm{T}(\mathrm{H}) 2$ paradigm. Nat Rev Immunol 2004; 4: 583-94.

3 Wynn TA. Common and unique mechanisms regulate fibrosis in various fibroproliferative diseases. J Clin Invest 2007; 117: 524-9.

4 Reiman RM, Thompson RW, Feng CG, Hari D, Knight R, Cheever AW, et al. Interleukin-5 (IL-5) augments the progression of liver fibrosis by regulating IL-13 activity. Infect Immun 2006; 74: 1471-9.

5 Weng H, Mertens PR, Gressner AM, Dooley S. IFN-gamma abrogates profibrogenic TGF-beta signaling in liver by targeting expression of inhibitory and receptor Smads. J Hepatol 2007; 46: 295-303.

6 Berre LL, Herve C, Buzelin F, Usal C, Soulillou JP, Dantal J. Renal macrophage activation and Th2 polarization precedes the development of nephrotic syndrome in Buffalo//Mna rats. Kidney Int 2005; 68: 2079-90.

7 Mills CD, Kincaid K, Alt JM, Heilman MJ, Hill AM. M-1/M-2 macrophages and the Th1/Th2 paradigm. J Immunol 2000; 164: 6166-73.

8 Meneghin A, Hogaboam CM. Infectious disease, the innate immune response, and fibrosis. J Clin Invest 2007; 117: 530-8.

9 Tipping PG. Toll-like receptors: the interface between innate and adaptive immunity. J Am Soc Nephrol 2006; 17: 1769-71.

10 Bhattacharjee RN, Akira S. Modifying toll-like receptor 9 signaling for therapeutic use. Mini Rev Med Chem 2006; 6: 287-91.

11 Krieg AM. Therapeutic potential of Toll-like receptor 9 activation. Nat Rev Drug Discov 2006; 5: 471-84.

12 Katakura K, Lee J, Rachmilewitz D, Li G, Eckmann L, Raz E. Toll-like receptor 9-induced type I IFN protects mice from experimental colitis. J Clin Invest 2005; 115: 695-702.

13 Suzuki H, Suzuki Y, Narita I, Aizawa M, Kihara M, Yamanaka T, et al. Toll-like receptor 9 affects severity of IgA nephropathy. J Am Soc Nephrol 2008; 19: 2384-95.

14 Christensen SR, Shupe J, Nickerson K, Kashgarian M, Flavell RA, Shlomchik MJ. Toll-like receptor 7 and TLR9 dictate autoantibody specificity and have opposing inflammatory and regulatory roles in a murine model of lupus. Immunity 2006; 25: 417-28.

15 Sato M, Muragaki Y, Saika S, Roberts AB, Ooshima A. Targeted disruption of TGF-beta1/Smad3 signaling protects against renal tubulointerstitial fibrosis induced by unilateral ureteral obstruction. J Clin Invest 2003; 112: 1486-94.

16 Vielhauer V, Anders HJ, Mack M, Cihak J, Strutz F, Stangassinger M, et al. Obstructive nephropathy in the mouse: progressive fibrosis correlates with tubulointerstitial chemokine expression and accumulation of CC chemokine receptor 2- and 5-positive leukocytes. J Am Soc Nephrol 2001; 12: 1173-87.

17 Hinz B, Phan SH, Thannickal VJ, Galli A, Bochaton-Piallat ML, Gabbiani G. The myofibroblast: one function, multiple origins. Am J Pathol 2007; 170: 1807-16.

18 Dantas M, Romão EA, Costa RS, dos Reis MA, Vieira Neto OM, Ribeiro $\mathrm{RA}$, et al. Urinary excretion of monocyte chemoattractant protein-1: a biomarker of active tubulointerstitial damage in patients with glomerulopathies. Kidney Blood Press Res 2007; 30: 306-13.

19 Prud'homme GJ. Pathobiology of transforming growth factor beta in cancer, fibrosis and immunologic disease, and therapeutic considerations. Lab Invest 2007; 87: 1077-91.

20 Oda T, Jung YO, Kim HS, Cai X, López-Guisa JM, Ikeda Y, et al. PAI-1 deficiency attenuates the fibrogenic response to ureteral obstruction. Kidney Int 2001; 60: 587-96.

21 Matsuo S, López-Guisa JM, Cai X, Okamura DM, Alpers CE, Bumgarner $\mathrm{RE}$, et al. Multifunctionality of PAl-1 in fibrogenesis: evidence from obstructive nephropathy in PAl-1-overexpressing mice. Kidney Int 2005; 67: 2221-38.

22 Wilson MS, Madala SK, Ramalingam TR, Gochuico BR, Rosas IO, Cheever AW, et al. Bleomycin and IL-1beta-mediated pulmonary fibrosis is IL-17A dependent. J Exp Med 2010; 207: 535-52.

23 O'Connor W Jr, Zenewicz LA, Flavell RA. The dual nature of $\mathrm{T}(\mathrm{H}) 17$ cells: shifting the focus to function. Nat Immunol 2010; 11: 471-6.

24 Thiery JP, Sleeman JP. Complex networks orchestrate epithelialmesenchymal transitions. Nat Rev Mol Cell Biol 2006; 7: 131-42.

25 Inazaki K, Kanamaru Y, Kojima Y, Sueyoshi N, Okumura K, Kaneko K, et al. Smad3 deficiency attenuates renal fibrosis, inflammation, and 
apoptosis after unilateral ureteral obstruction. Kidney Int 2004; 66: 597-604.

26 Schaier M, Vorwalder S, Sommerer C, Dikow R, Hug F, Gross ML, et al. Role of FTY720 on M1 and M2 macrophages, lymphocytes, and chemokines in 5/6 nephrectomized rats. Am J Physiol Renal Physiol 2009; 297: F769-80.

27 Conroy H, Marshall NA, Mills KH. TLR ligand suppression or enhancement of Treg cells? A double-edged sword in immunity to tumours. Oncogene 2008; 27: 168-80.

28 du Bois RM. Strategies for treating idiopathic pulmonary fibrosis. Nat Rev Drug Discov 2010; 9: 129-40.

29 Rockey DC. Current and future anti-fibrotic therapies for chronic liver disease. Clin Liver Dis 2008; 12: 939-62.

30 Oldroyd SD, Thomas GL, Gabbiani G, El Nahas AM. Interferon-gamma inhibits experimental renal fibrosis. Kidney Int 1999; 56: 2116-27.

31 Liu YY, Cai WF, Yang HZ, Cui B, Chen ZR, Liu HZ, et al. Bacillus Calmette-Guérin and TLR4 agonist prevent cardiovascular hypertrophy and fibrosis by regulating immune microenvironment. J Immunol 2008; 180: 7349-57.

32 Yang HZ, Cui B, Liu HZ, Chen ZR, Yan HM, Hua F, et al. Targeting TLR2 attenuates pulmonary inflammation and fibrosis by reversion of suppressive immune microenvironment. J Immunol 2009; 182: 692-702.

33 Hayashi T, Raz E. TLR9-based immunotherapy for allergic disease. Am J Med 2006; 119: 897.e1-6.

34 Ehlers M, Ravetch JV. Opposing effects of Toll-like receptor stimulation induce autoimmunity or tolerance. Trends Immunol 2007; 28: 74-9.

35 Sejima T, Madoiwa S, Mimuro J, Sugo T, Okada K, Ueshima S, et al. Protection of plasminogen activator inhibitor-1-deficient mice from nasal allergy. J Immunol 2005; 174: 8135-43.

36 Rincon M, Flavell RA, Davis RA. The JNK and p38 MAP kinase signaling pathways in T cell-mediated immune responses. Free Radic Biol Med 2000; 28: 1328-37.

37 Dong C, Davis RJ, Flavell RA. Signaling by the JNK group of MAP kinases. J Clin Immunol 2001; 21: 253-7.

$38 \mathrm{Yu} \mathrm{H}$, Kortylewski M, Pardoll D. Crosstalk between cancer and immune cells: role of STAT3 in the tumour microenvironment. Nat Rev Immunol 2007; 7: 41-51.

39 Anders HJ, Vielhauer V, Eis V, Linde Y, Kretzler M, Perez de Lema G, et al. Activation of toll-like receptor-9 induces progression of renal disease in MRL-Fas(Ipr) mice. FASEB J 2004; 18: 534-6. 